\title{
Performance Investigation of MIMO-OFCDM with Imperfect Channel Estimation
}

\author{
Yiqing $\mathrm{Zhou}^{\# 1}$ and Tung-Sang $\mathrm{Ng}^{{ }^{2}}$ \\ ${ }^{\#}$ Hong Kong Applied Science and Technology Research Institute Company Limited \\ 1yqzhou@astri.org \\ ${ }^{*}$ Dept. of Electrical and Electronic Engineering, University of Hong Kong \\ Pokfulam Road, Hong Kong, P.R. China \\ ${ }^{2}$ tsng@eee.hku.hk
}

\begin{abstract}
This paper investigates the performance of multiple input multiple output orthogonal frequency and code division multiplexing (MIMO-OFCDM), where practical channel estimation is carried out with time-multiplexed pilot signals. Conditioned on each channel realization and estimation, the BER of MIMO-OFCDM is analytically evaluated, assuming zeroforcing successive interference cancellation (ZF-SIC) in the space domain and minimum mean square error (MMSE) detection in the frequency domain. Then, the conditional BER is averaged over all channel realizations by means of simulations. It is shown that with various channel estimation qualities and channel correlation conditions, the proposed semi-analytical approach provides precise performance estimations.
\end{abstract}

Keywords- MIMO, OFCDM, Two-dimensional spreading, channel estimation, multi-code transmission

\section{INTRODUCTION}

Wireless access technologies are developing rapidly from frequency domain multiplexing access (FDMA), time domain multiplexing access (TDMA), code domain multiplexing access (CDMA) [1] to the most recent orthogonal frequency division multiplexing (OFDM). Combining OFDM with twodimensional (2-D) spreading [2], orthogonal frequency and code division multiplexing (OFCDM) [3] has been proposed as a promising wireless access technique for the downlink transmission in future wireless communications. Based on OFDM, OFCDM can combat the severe multipath interference in a broadband channel. Moreover, using 2-D spreading, each data symbol in the OFCDM system is spread in time domain with $N_{T}$ chips and in frequency domain with $N_{F}$ chips. Given a 2-D spreading of $N=N_{T} \times N_{F}$, there are up to $N$ different orthogonal 2-D code channels. Thus, multi-code transmission can be employed to increase the data rate.

Multiple-input and multiple-output (MIMO) multiplexing is widely taken as an effective technique to provide high speed transmissions in future broadband wireless communications. Although MIMO-OFDM has been widely investigated, there are only a few studies on MIMO-OFCDM. This paper aims to investigate the performance of MIMO-OFCDM with imperfect channel estimation. Pilot signals are timemultiplexed to data signals and used for channel estimation. In the multi-code MIMO-OFCDM system, desired data signals are interfered by not only co-channel signals from other transmit antennas (or multi-antenna interference (MTI)), but also multi-code interference (MCI) [4] from other code channels. Therefore, zero-forcing successive interference cancellation (ZF-SIC) is employed in the space domain to combat MTI and minimum mean square error (MMSE) detection is used in the frequency domain to suppress MCI. A performance analysis is then carried out for uncoded MIMOOFCDM. At each step of SIC, given channel estimation, an accurate analytical BER can be obtained by taking the correlation between error events into account. Then, the conditional BER is averaged over all channel realizations to get the final BER. It is shown that with various channel estimation qualities and channel correlation conditions, the proposed semi-analytical approach provides precise performance estimations.

The rest of the paper is organized as follows. Section II describes the system model of MIMO-OFCDM. Section III presents the semi-analytical approach. Then numerical and simulation results are shown in Section IV. Finally, conclusions are drawn in the last section.

\section{SYSTEM DESCRIPTION}

\section{A. Transmitter}

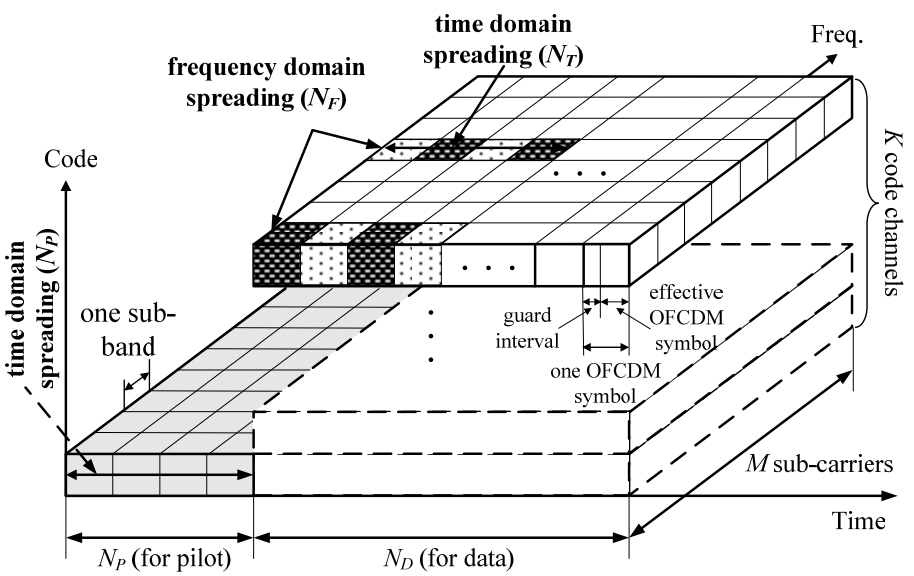

Fig. 1. Signal packet structure at each antenna

The MIMO-OFCDM system transmits independent data sub-streams at different antennas in the same frequency band simultaneously. Information bits are firstly serial to parallel 
(S/P) converted into $n_{t}$ sub-streams, corresponding to $n_{t}$ transmit antennas. For each antenna, the sub-stream is channel coded and processed by the 2-D spread OFCDM and multicode transmission with $K$ data code channels [5]. The 2-D spread signals from the $K$ code channels are added together at the code multiplexer and pass through a chip interleaver in the frequency domain. At the same time, known pilot symbols are $\mathrm{S} / \mathrm{P}$ converted into $M$ sub-streams, spread in the time domain, and time-multiplexed with data signals. The resultant signals are then processed by the IFFT and the guard interval insertion. The signal packet structure at each antenna is shown in Fig. 1. It can be seen that in the time domain, the packet starts with $N_{P}$ OFCDM symbols for pilot, followed by $N_{D}$ symbols for data transmission. The duration of each OFCDM symbol is $T_{s}=T_{e}+T_{g}$, with $T_{e}$ and $T_{g}$ denoting the effective OFCDM symbol duration and guard interval, respectively. In the frequency domain, there are totally $M$ sub-carriers and pilot symbols are adopted at each sub-carrier. Finally, in the code domain, only one time domain spreading code is used by the pilot channel, while $K$ 2-D codes are employed for data transmission. Accordingly, the signal transmitted during the $i^{\text {th }}$ OFCDM symbol on the $m^{\text {th }}$ sub-carrier at the $p^{\text {th }}$ $\left(0 \leq p \leq n_{t}-1\right)$ antenna is given by

$$
s_{p, m, i}=\left\{\begin{array}{ll}
d_{p i l o t, p, m} c_{N_{P}, i}^{(p)} & 0 \leq i \leq N_{P}-1 \\
\sum_{k=0}^{K-1} d_{p, i, m, k} C_{i, m, k} & N_{P} \leq i \leq N_{P}+N_{D}-1
\end{array},\right.
$$

where $d_{p i l o t, p, m}$ is the pilot symbol with a power of $\beta P_{d}, d_{p, i, m, k}$ is the data symbol of the $k^{\text {th }}$ code channel with a power of $P_{d}$, and $c_{N_{P}, i}^{(p)}$ and $C_{i, m, k}$ are the spreading code for the pilot and data channel, respectively.

\section{B. Channel Estimation}

Assuming a slow fading channel where the fading factor is fixed in one packet duration, $h_{q, p, m}$ can be used to denote the complex channel fading on the $m^{\text {th }}$ sub-carrier from the $p^{\text {th }}$ transmit antenna to the $q^{\text {th }}$ receive antenna. The amplitude and phase of $h_{q, p, m}$ are assumed to be Rayleigh distributed with $P_{c h}=E\left\{\left|h_{q, p, m}\right|^{2}\right\}$ and uniformly distributed in $[0,2 \pi)$, respectively. Furthermore, the sub-carrier correlation coefficient in the frequency domain between $h_{q, p, m_{0}}$ and $h_{q, p, m_{1}}$ is given by

$$
\begin{aligned}
\rho_{m_{0}, m_{1}} & =\frac{E\left\{h_{q, p, m_{0}}^{*} h_{q, p, m_{1}}\right\}}{\sqrt{E\left\{\left|h_{q, p, m_{0}}\right|^{2}\right\}} \sqrt{E\left\{\left|h_{q, p, m_{0}}\right|^{2}\right\}}} \\
& =\frac{1}{\sqrt{1+\left(\Delta f / \Delta f_{c}\right)^{2}}},
\end{aligned}
$$

where $\Delta f$ is the frequency separation between the $m_{0}^{\text {th }}$ and $m_{1}^{\text {th }}$ sub-carriers and $\Delta f_{c}$ is the coherence bandwidth of the channel. For different transmit or receive antennas, the channel fading is assumed to be independent to each other.

At the receiver, after the FFT on the $\bar{q}$ th $\left(0 \leq \bar{q} \leq n_{r}-1\right)$ receive antenna, the signals on the $\bar{m}^{\text {th }}$ sub-carrier is given by

$$
y_{\bar{q}, \bar{i}, \bar{m}}=\sum_{p=0}^{n_{t}-1} h_{\bar{q}, p, \bar{m}} d_{p i l o t, p, \bar{m}} c_{N_{P}, \bar{i}}^{(p)}+\eta_{\bar{q}, \bar{i}, \bar{m}}
$$

for $0 \leq \bar{i} \leq N_{P}-1$, and

$$
y_{\bar{q}, \bar{i}, \bar{m}}=\sum_{p=0}^{n_{t}-1} h_{\bar{q}, p, \bar{m}} \sum_{k=0}^{K-1} d_{p, \bar{i}, \bar{m}, k} C_{\bar{i}, \bar{m}, k}+\eta_{\bar{q}, \bar{i}, \bar{m}}
$$

for $N_{P} \leq \bar{i} \leq N_{d}-1$, where $\eta_{\bar{q}, \bar{i}, \bar{m}}$ is the AWGN noise with zero mean and $\sigma_{n}^{2}$ variance. The first $N_{P}$ signals carry pilot symbols and are used for channel estimation. On the $\bar{m}^{\text {th }}$ subcarrier, the channel factor from the $\bar{p}^{\text {th }}$ transmit antenna to the $\bar{q}^{\text {th }}$ receive antenna is given by

$$
\begin{aligned}
\hat{h}_{\bar{q}, \bar{p}, \bar{m}} & =\left(d_{\text {pilot }, \bar{p}}\right)^{-1}\left(\sum_{\bar{i}=0}^{N_{P}-1} y_{\bar{q}, \bar{i}, \bar{m}} c_{N_{P}, \bar{i}}^{(\bar{p})} / N_{P}\right) \\
& =h_{\bar{q}, \bar{p}, \bar{m}}+v_{\bar{q}, \bar{p}, \bar{m}}
\end{aligned}
$$

where $v_{\bar{q}, \bar{p}, \bar{m}}$ is the noise with zero mean and variance of $\sigma_{H}^{2}=\sigma_{n}^{2} /\left(\beta P_{d} N_{P}\right)$.

\section{Signal Detection}

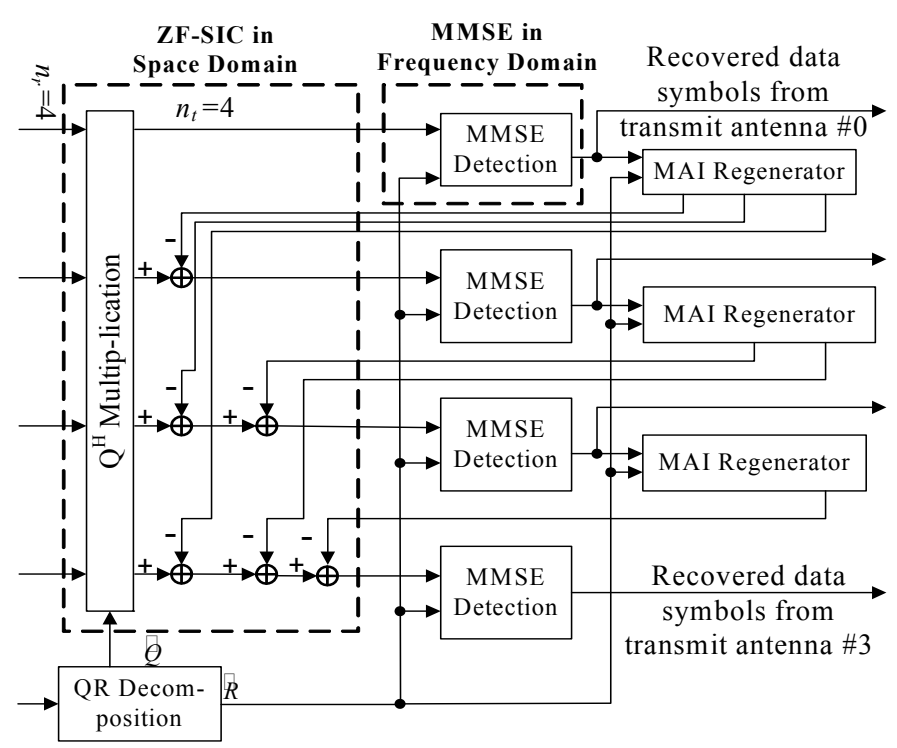

Figure 2. Signal detection with ZF-MMSE-SIC at the receiver

On each sub-carrier, the data signals are processed by the time domain despreader to collect the useful signals in the time domain and the resultant signal is denoted as $\bar{y}_{q, \bar{m}}$. Then, as illustrated in Fig. 2, a ZF-SIC with QR decomposition is employed in the space domain to combat MTI. The QR decomposition of the estimated channel matrix $\hat{H}_{\bar{m}}$ is given 
by $\hat{H}_{\bar{m}}=\hat{Q}_{\bar{m}} \hat{R}_{\bar{m}}$. After multiplying $\bar{Y}_{\bar{m}}=\left(\bar{y}_{0, \bar{m}}, \cdots, \bar{y}_{n_{r}-1, \bar{m}}\right)^{T}$ with $\hat{Q}_{\bar{m}}^{H}$, the signal is given by

$$
\begin{aligned}
\bar{\Upsilon}_{\bar{m}} & =\hat{Q}_{\bar{m}}^{H} \bar{Y}_{\bar{m}}=\hat{Q}_{\bar{m}}^{H}\left(\hat{H}_{\bar{m}}+v_{\bar{m}}\right) \bar{D}_{\bar{m}}^{-}+\hat{Q}_{\bar{m}}^{H} \overline{\mathrm{N}}_{\bar{m}} \\
& =\hat{R}_{\bar{m}} \bar{D}_{\bar{m}}^{-}+\Delta_{\bar{m}}
\end{aligned}
$$

where $\bar{D}_{\bar{m}}^{\bar{m}}=\left(\sum_{k=0}^{K_{C}} d_{0, k} c_{N_{F}, \bar{m}}^{(k)}, \cdots, \sum_{k=0}^{K_{C}} d_{n_{t}-1, k} c_{N_{F}, \bar{m}}^{(k)}\right)^{T}, v_{\bar{m}}$ is a $n_{r} \times n_{t}$ channel estimation noise matrix, $\overline{\mathrm{N}}_{\bar{m}}=\left(\bar{\eta}_{0, \bar{m}}, \cdots, \bar{\eta}_{n_{r}-1, \bar{m}}\right)^{T}$, and $\Delta_{\bar{m}}$ is the noise vector including the channel estimation error and background noise. Due to the upper triangular structure of $R_{\bar{m}}^{-}$, the $0^{\text {th }}$ element of $\bar{\Upsilon}_{\bar{m}}^{-}$is free of MTI, but there is MCI because the same set of data symbols $\sum_{k=0}^{K_{C}} d_{0, k}$ are impressed on $N_{F}$ interleaved sub-carriers. Thus, MMSE detection is employed in the frequency domain to recover the data symbol on each code channel. After MMSE, an estimation of the MTI caused by the $0^{\text {th }}$ transmitting antenna is generated and MTI cancellation can be carried out on the $1^{\text {st }}, 2^{\text {nd }}, \ldots$, and $\left(n_{t}-1\right)^{\text {th }}$ antennas. In summary, after the MTI cancellation at the $s^{\text {th }}$ step of ZF-MMSE-SIC, the resultant signal is given by

$$
\begin{aligned}
\gamma_{s, \bar{m}}^{(s)} & =\gamma_{s, \bar{m}}-\left(\sum_{p=0}^{s-1} \hat{r}_{m, p, s}^{-} \sum_{k=0}^{K_{C}} \hat{d}_{p, k} c_{N_{F}, \bar{m}}^{(k)}\right) \\
& =\left(\hat{r}_{\bar{m}, s, s}^{-} \sum_{k=0}^{K_{C}} d_{s, k} c_{N_{F}, \bar{m}}^{(k)}\right)+\eta_{s, \bar{m}} \\
& +\underbrace{\sum_{p=s+1}^{n_{t}-1} \hat{r}_{m, p, s}^{-} \sum_{k=0}^{K_{C}}\left(d_{p, k}-\hat{d}_{p, k}\right) c_{N_{F}, \bar{m}}^{(k)}}_{M T I_{s, \bar{m}}}
\end{aligned}
$$

where $\hat{d}_{p, k}$ is the data decision on the $p^{\text {th }}$ antenna and $M T I_{s, \bar{m}}$ is the residual MTI. Then, the MMSE weight is given by

$$
\begin{aligned}
l_{s, \bar{m}} & =\frac{P_{d} \cdot \hat{r}_{m, s, s}^{-} c_{N_{F}, \bar{m}}^{(\bar{k})}}{P_{d}\left(K_{C}+1\right) \hat{r}_{m, s, s}^{2}+E\left\{\left|M T I_{s, \bar{m}}\right|^{2}\right\}+\sigma^{2}} \\
& =\omega_{s, \bar{m}} c_{N_{F}, \bar{m}}^{(\bar{k})} .
\end{aligned}
$$

where $\sigma^{2}$ is the Gaussian noise including channel estimation error and background noise.

The output of MMSE detection is further normalized by $\lambda_{s}=1 / \sum_{\bar{m}} \omega_{s, \bar{m}} \hat{r}_{\bar{m}, 0,0}$ to simplify the notation in performance analysis. Finally, the decision variable is given by

$$
\begin{aligned}
y_{s, \bar{k}} & =\underbrace{d_{s, \bar{k}}}_{N o i_{s, \bar{k}}}+\underbrace{\lambda_{s} \sum_{\bar{m}} \omega_{s, \bar{m}} c_{N_{F}, \bar{m}}^{(\bar{k})}\left[\sum_{p=0}^{s-1} \hat{r}_{\bar{m}, p, s} \sum_{k=0}^{K_{C}}\left(d_{p, k}-\hat{d}_{p, k}\right) c_{N_{F}, \bar{m}}^{(k)}\right]}_{M I_{s} \sum_{\bar{m}} \omega_{s, \bar{m}} c_{N_{F}, \bar{m}}^{(\bar{k})} \eta_{s, \bar{m}}} \\
& +\underbrace{\lambda_{s} \sum_{\bar{m}} \sum_{k=0, k \neq \bar{k}}^{K_{C}} \omega_{s, \bar{m}} c_{N_{F}, \bar{m}}^{(\bar{k})} \hat{r}_{\bar{m}, s, s} d_{s, k} c_{N_{F}, \bar{m}}^{(k)}}_{M C I_{s, \bar{k}}}
\end{aligned}
$$

\section{PERFORMANCE ANALYSIS}

\section{A. BER at the $0^{\text {th }}$ step}

Assuming QPSK modulation, the BER of the real part of $y_{0, \bar{k}}$, i.e., $y_{I, 0, \bar{k}}$, is considered. Given the estimated channel matrix on $\quad N_{F} \quad$ sub-carriers $\quad \hat{\mathbf{H}}=\left\{\hat{H}_{0}, \hat{H}_{M / N_{F}}, \cdots, \hat{H}_{M-M / N_{F}}\right\} \quad$ and approximating $\mathrm{MCI}$ as a Gaussian variable, the conditional BER at the $0^{\text {th }}$ step of ZF-MMSE-SIC is given by

$P_{e, \hat{\mathbf{H}}}^{(0)}=\operatorname{Pr}\left(\hat{d}_{I, 0, \bar{k}} \neq d_{I, 0, \bar{k}} \mid \hat{\mathbf{H}}\right)=Q\left(\sqrt{P_{d} /\left(2 \sigma_{I}^{2}(0)\right)}\right)$,

where $\sigma_{I}^{2}(0)=\sigma_{I, M C I, \bar{k}}^{2}(0)+\sigma_{I, N o i, \bar{k}}^{2}(0)$ is the sum of the variances of $\mathrm{MCI}$ and noise and $Q(x)$ is the Q-function. $\sigma_{I, M C I, \bar{k}}^{2}(0)$ and $\sigma_{I, N o i, \bar{k}}^{2}(0)$ can be obtained from

$$
\left\{\begin{array}{l}
\sigma_{I, M C I, \bar{k}}^{2}(0)=\frac{P_{d}}{2} \lambda_{0}^{2} \sum_{k=0, k \neq \bar{k}}^{K_{C}}\left|\sum_{\bar{m}} \omega_{0, \bar{m}} \hat{r}_{m, 0,0}^{-} c_{N_{F}, \bar{m}}^{(k)} c_{N_{F}, \bar{m}}^{(\bar{k})}\right|^{2} \\
\sigma_{I, N o i, \bar{k}}^{2}(0)=\frac{\sigma^{2}}{2} \lambda_{0}^{2} \sum_{\bar{m}}\left|\omega_{0, \bar{m}}\right|^{2}
\end{array}\right.
$$

\section{B. BER at the sth step}

Given channel estimation $\hat{\mathbf{H}}$, the conditional BER at the $s^{\text {th }}$ step is expressed as

$$
\begin{aligned}
& P_{e, \hat{\mathbf{H}}}^{(s)}=\operatorname{Pr}\left(\hat{d}_{I, s, \bar{k}} \neq d_{I, s, \bar{k}} \mid \hat{\mathbf{H}}\right) \\
& =\sum_{N_{e, 0}=0}^{2 K_{c}+2} \cdots \sum_{N_{e, s-1}=0}^{2 K_{c}+2}\left[\begin{array}{l}
\operatorname{Pr}\left(\hat{d}_{I, s, \bar{k}} \neq d_{I, s, \bar{k}} \mid \hat{\mathbf{H}},\left\{N_{e, 0}, \cdots, N_{e, s-1}\right\}\right) \\
\cdot \operatorname{Pr}\left(N_{e, 0}, \cdots, N_{e, s-1} \mid \hat{\mathbf{H}}\right)
\end{array}\right]
\end{aligned}
$$

where $d_{I, s, \bar{k}}$ is the real part of $d_{s, \bar{k}}, 2 K_{C}+2$ is the maximum number of bit errors at each step, and $\operatorname{Pr}\left(N_{e, 0}, \cdots, N_{e, s-1} \mid \hat{\mathbf{H}}\right)$ denotes the probability of the event that there are $N_{e, 0}, \ldots$, 
$N_{e, s-1}$ bit errors at the $0^{\text {th }}, \ldots,(s-1)^{\text {th }}$ step, respectively.

First of all, by taking the interference and noise terms, i.e., $M T I_{s, \bar{k}}, M C I_{s, \bar{k}}$ and $\mathrm{Noi}_{s, \bar{k}}$, in the decision variable (8) as three independent Gaussian distributed variables, it can be obtained that

$\operatorname{Pr}\left(\hat{d}_{I, s, \bar{k}} \neq d_{I, s, \bar{k}} \mid \hat{\mathbf{H}},\left\{N_{e, 0}, \cdots, N_{e, s-1}\right\}\right)=Q\left(\sqrt{P_{d} /\left(2 \sigma_{I, \bar{k}}^{2}(s)\right)}\right)$,

where $\sigma_{I, \bar{k}}^{2}(s)$ is the sum of the variance of $M T I_{s, \bar{m}}, M C I_{s, \bar{k}}$, and $\mathrm{Noi}_{s, \bar{k}}$.

Secondly, the joint event probability $\operatorname{Pr}\left(N_{e, 0}, \cdots, N_{e, s-1} \mid \hat{\mathbf{H}}\right)$ can be obtained a series of single event probabilities

$$
\begin{gathered}
\operatorname{Pr}\left(N_{e, 0}, \cdots, N_{e, s-1} \mid \hat{\mathbf{H}}\right)=\operatorname{Pr}\left(N_{e, s-1} \mid N_{e, 0}, \cdots, N_{e, s-2}, \hat{\mathbf{H}}\right) \\
\cdot \operatorname{Pr}\left(N_{e, s-2} \mid N_{e, 0}, \cdots, N_{e, s-3}, \hat{\mathbf{H}}\right) \cdots \\
\cdot \operatorname{Pr}\left(N_{e, 1} \mid N_{e, 0}, \hat{\mathbf{H}}\right) \cdot \operatorname{Pr}\left(N_{e, 0} \mid \hat{\mathbf{H}}\right) .
\end{gathered}
$$

Since $N_{e, 0}$ is the total number of bit errors at the $0^{\text {th }}$ step on all $K_{C}+1$ code channels, $\operatorname{Pr}\left(N_{e, 0} \mid \hat{\mathbf{H}}\right)$ is calculated by enumerating over all types of error events happened on code channels making the sum of bit errors equal to $N_{e, 0}[6]$. Note that for different code channels at the same step, the MCI (or noise) terms in the decision variable of real part are correlated to each other, i.e., $\alpha_{M C I}\left(k_{0}, k_{1}\right)=E\left\{M C I_{I, 0, k_{0}} M C I_{I, 0, k_{1}}\right\}$ is not zero. Therefore, the decision variables on the $K_{C}+1$ code channels are correlated. Hence, the calculation of $\operatorname{Pr}\left(N_{e, 0} \mid \hat{\mathbf{H}}\right)$ involves a multivariate Gaussian distribution of $K_{C}+1$ dimensions, which becomes very complex when $K_{C}+1$ is large. One method to tackle this problem is to ignore the correlation between error events, but this approximation results in an underestimation of the BER. To reduce the complexity and take the correlations into account, another approach is to approximate the multivariate Gaussian probability by a group of two-variate probabilities, which can be upper bounded [7] and make the calculation feasible. As verified by simulations, this approach can provide more accurate estimation on the BER performance than the one ignoring correlations.

After calculating $\operatorname{Pr}\left(N_{e, 1} \mid N_{e, 0}, \hat{\mathbf{H}}\right)$ etc. in a similar way as that of $\operatorname{Pr}\left(N_{e, 0} \mid \hat{\mathbf{H}}\right), \operatorname{Pr}\left(N_{e, 0}, \cdots, N_{e, s-1} \mid \hat{\mathbf{H}}\right)$ and thus $P_{e, \mathbf{H}}^{(s)}$ can be obtained. Finally, $P_{e, \mathbf{H}}^{(s)}$ is averaged over all $\hat{\mathbf{H}}$ to obtain the final average BER at the $s^{\text {th }}$ step, which can be numerically evaluated by a Monte Carlo Approach.

\section{NUMERICAL RESULTS}

Some representative numerical and simulation results are shown in this section. The configuration of the multi-code MIMO-OFCDM system is as follows. The number of transmit antennas and receive antennas are both four. The system bandwidth is set to $100 \mathrm{MHz}$ and the number of sub-carriers $M$ is 1024. The time domain spreading factor $N_{T}$ equals eight and the frequency domain spreading factor $N_{F}$ is 16 . To obtain the maximum data rate, a full loaded MIMO-OFCDM system with $K=N$ data code channels is considered. Each packet is composed of $N_{d}=52$ OFCDM symbols, with the first $N_{P}=4$ symbols reserved as pilot symbols.

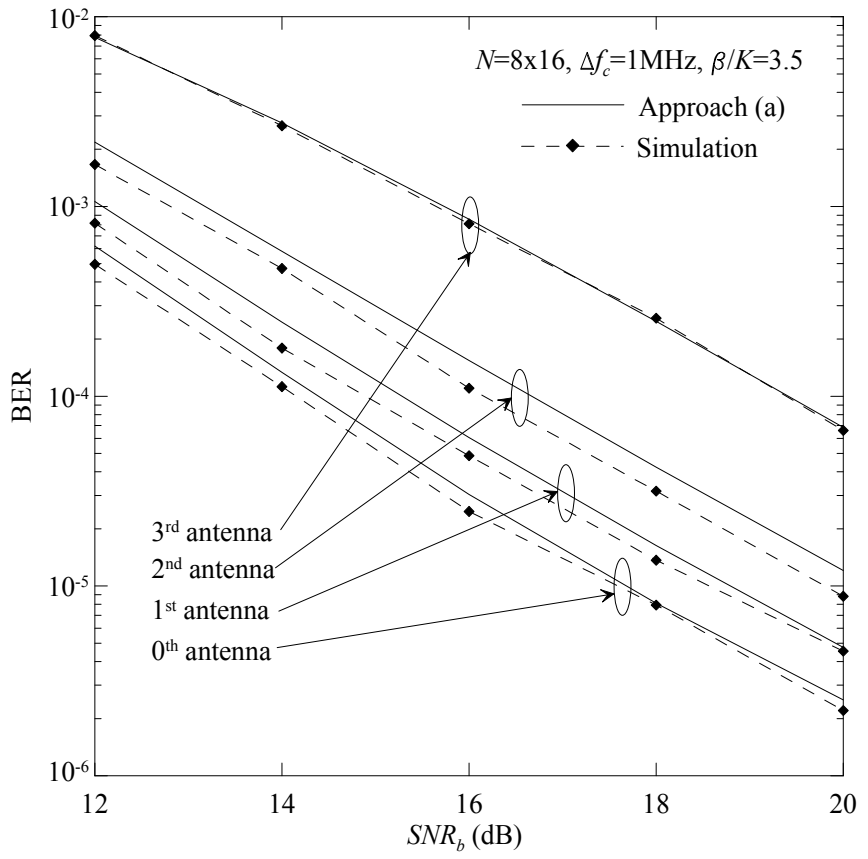

Figure 3(a). System performance as a function of $S N R_{b}$

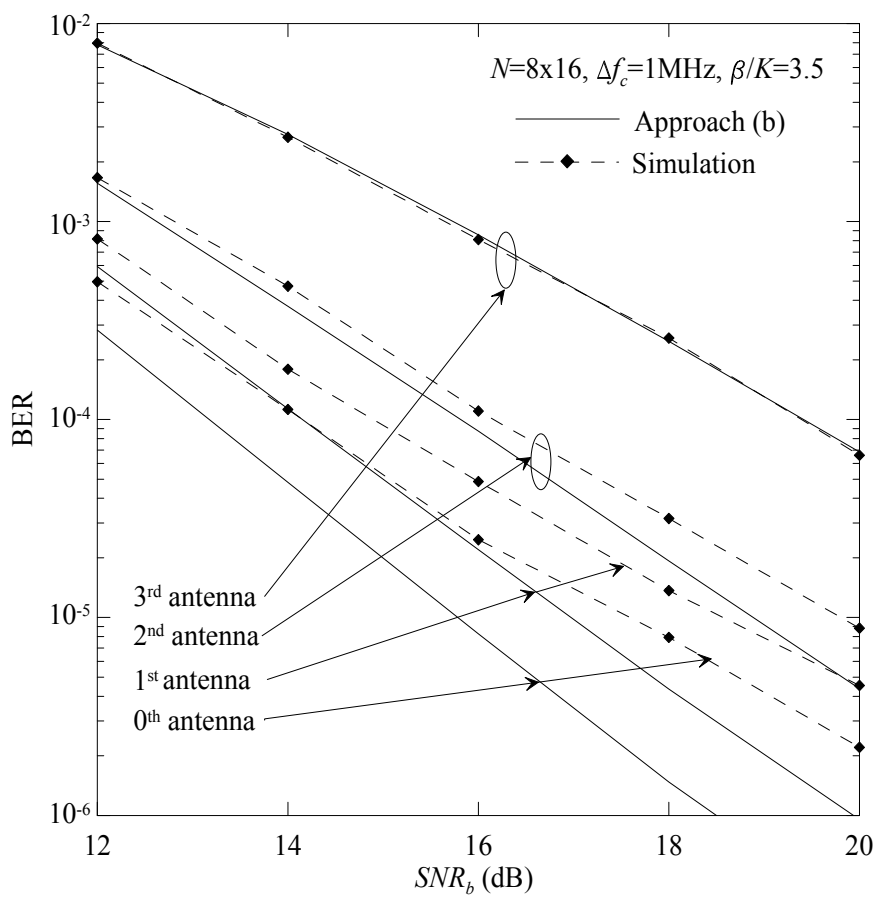

Figure 3(b). System performance as a function of $S N R_{b}$ 
The semi-analytical performance of an uncoded system is verified by simulations in Fig. 3 as a function of SNR. As a comparison, Fig. 3(a) and 3(b) plot the BERs calculated from the proposed analytical approach (approach (a)) and the one where no correlation is considered (approach (b)), respectively. It can be seen that for the $3^{\text {rd }}$ antenna, the analytical results of both approaches match well with the simulated ones, because at the $0^{\text {th }}$ step of ZF-MMSE-SIC detection there is no MAI cancellation and no error propagation. For later detected antennas, the analytical results of approach (a) keep close to the simulated BER, while the results of approach (b) deviate from the simulated ones, especially at high SNR. The reason is that as the ZF-MMSE-SIC detection goes on step by step, the errors at previous steps propagate to the current one and have a cumulative effect on the BER performance. Their influence is apparent in the $2^{\text {nd }}$ and $3^{\text {rd }}$ steps as they dominate the BER performance, particularly at high SNR. It is clear that the correlation between the error events at previous steps should be taken into account, otherwise the analytical BER becomes too optimistic.

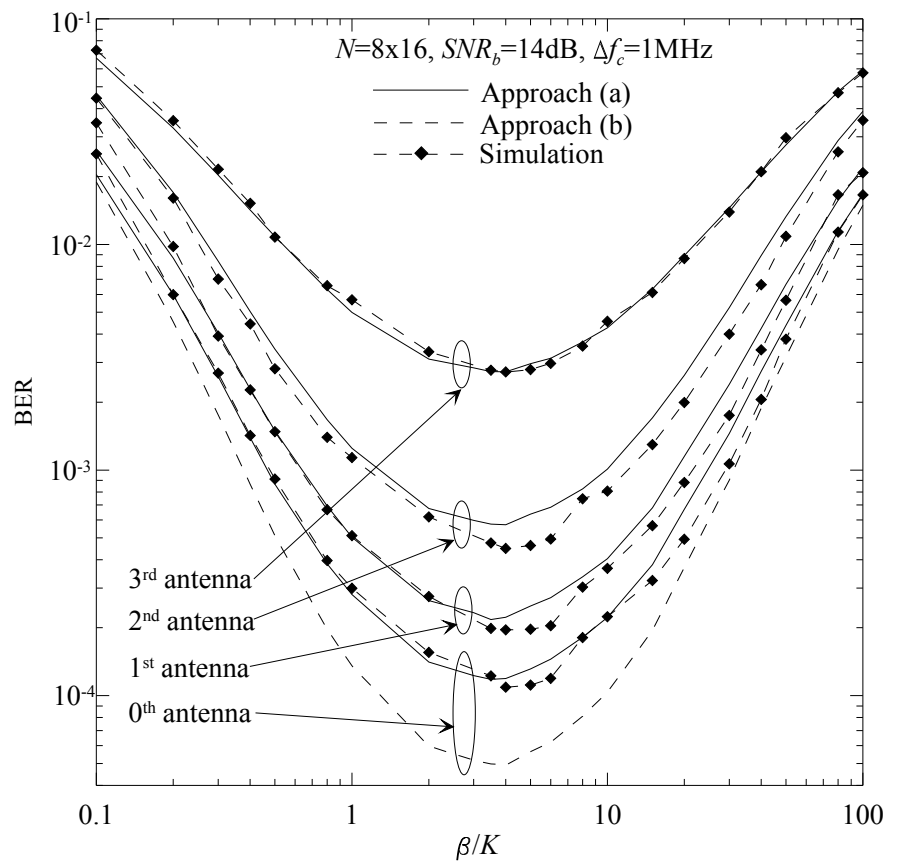

Figure 4. System performance as a function of power ratio

For the sake of clarity, the analytical results of approach (b) are only shown for the last stage of detection in the following investigations. As illustrated in Section II, the MIMO-OFCDM system employs a practical channel estimation algorithm by using the time-multiplexed pilot channel. Since the total transmission power is limited, the power should be suitably allocated to the data channels and the pilot channel to achieve the best performance. Fig. 4 shows the system performance as a function of the power ratio between the pilot and all data channels $(\beta / K)$. It can be seen that when $\beta / K$ is small, the pilots have low power and the channel estimation quality is poor, resulting in a high BER.
When $\beta / K$ increases, the BER reduces as the quality of channel estimation improves. The BER reaches a minimum value for a particular value of $\beta / K$. Further increasing $\beta / K$ beyond that value increases BER due to too little power assigned to data channels. At different antennas, the system always achieves near optimum performance when $\beta / K$ takes value between 2.0 to 6.0 . It can also be seen that the analytical results obtained from the proposed approach matches well with the simulations for all values of $\beta / K$, while the analytical BER of approach (b) for the last stage of detection is much lower than the simulated BER, especially near the optimum $\beta / K$. This demonstrates that under various channel estimation qualities, the proposed semi-analytical approach can provide accurate performance evaluation.

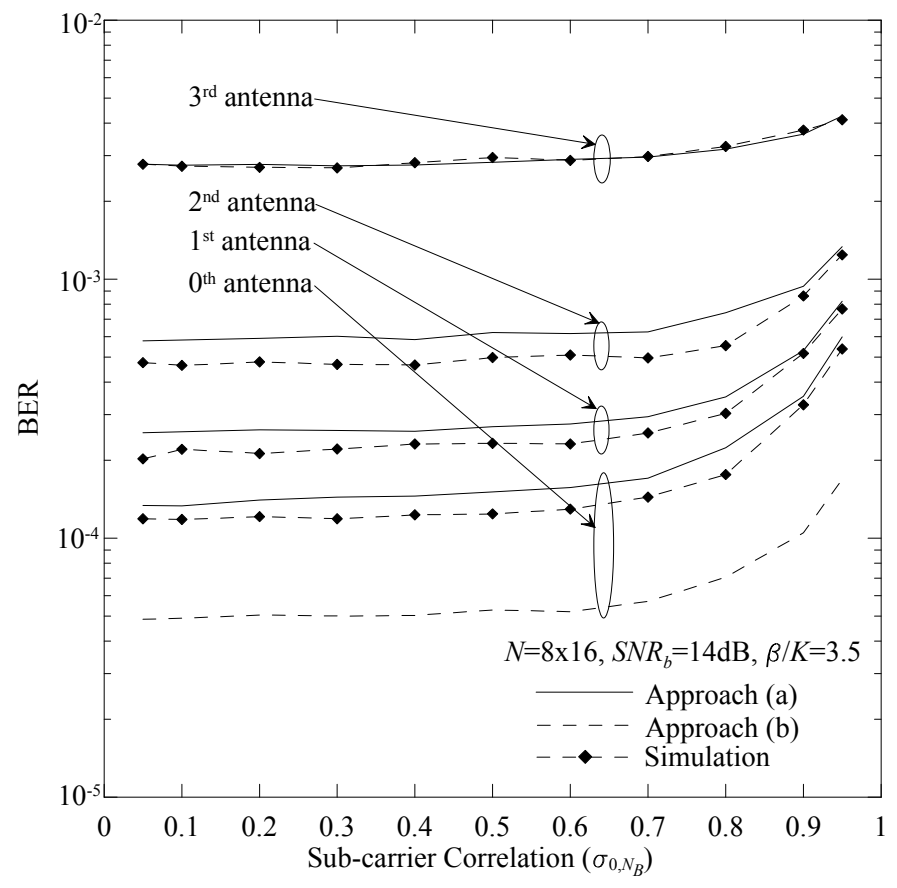

Figure 5. System performance with different sub-carrier correlations

Fig. 5 illustrates the effect of sub-carrier correlation on the BER performance. Given a system bandwidth, the number of sub-carriers $M$ and spreading scheme $N=N_{T} \times N_{F}$, the correlation coefficient $\rho_{0, N_{B}}\left(N_{B}=M / N_{F}\right)$ varies with channel coherence bandwidth $\Delta f_{c}$. Again, approach (a) provides precise BER estimation with various sub-carrier correlation conditions, while the BER on the last stage of detection using approach (b) is significantly underestimated. Moreover, it can be seen that the BER increases with $\rho_{0, N_{B}}$. This is because frequency diversity gain can be obtained in the MIMO-OFCDM system by means of a spreading of $N_{F}=16$ in the frequency domain and MMSE detection. When $\rho_{0, N_{B}}$ gets larger, the frequency diversity gain reduces, resulting in a 
degraded BER. However, the BER degradation of MIMOOFCDM is negligible for $\rho_{0, N_{B}} \leq 0.7$.

\section{CONCLUSIONS}

With practical channel estimation, a MIMO-OFCDM system with 2-D spreading and multi-code transmission has been semi-analytically investigated in this paper. The semianalytical results have been verified by simulations. It can be concluded that under various channel estimation qualities and channel correlation conditions, the proposed semi-analytical approach provided a precise estimation for the system performance which was more accurate than the one considering no correlation.

\section{ACKNOWLEDGMENT}

This work was supported by The University of Hong Kong Research Committee.

\section{REFERENCES}

[1] J. Wang and L. B. Milstein, "CDMA overlay situations for microcellular mobile communications," IEEE Trans. Commun., vol. 43, no. 2/3/4, pp. 603-614, Feb/March/April 1995.

[2] Anders Persson, Tony Ottosson, and Erik Strom, "Time-frequency localized CDMA for downlink multi-carrier systems," IEEE ISSSTA 2002, pp. 118-122, Sept. 2002.

[3] H. Atarashi, S. Abeta, and M. Sawahashi, "Broadband packet wireless access appropriate for high-speed and high-capacity throughput," IEEE VTC2001-Spring, pp. 566-570, May 2001.

[4] J. Chen, J. Wang and M. Sawahashi, "MCI cancellation for multi-code wideband CDMA," IEEE Journal on Selected Areas in Commun., vol. 20, no. 2, pp. 450-462, Feb. 2002.

[5] Y.Q. Zhou, J. Wang, T.S. Ng, K. Higuchi and M. Sawahashi, "OFCDM: a promising broadband wireless access technique," IEEE Commun. Magazine, vol. 46, pp. 39-49, March 2008.

[6] Y.Q. Zhou, J. Wang, and M. Sawahashi, "Downlink Transmission of Broadband OFCDM Systems---Part I: Hybrid Detection”, IEEE Trans. Commun., vol. 53, pp. 718-729, April 2005.

[7] A. H. Madsen and K. S. Cho, "MMSE/PIC multiuser detection for DS/CDMA systems with inter-and intra-cell interference", IEEE Trans.Commun., vol. 47, pp. 291-299, Feb. 1999. 